\title{
Calcium-modulated ciliary membrane guanylate cyclase transduction machinery: Constitution and operational principles
}

Teresa Duda, Ewa Fik-Rymarkiewicz, Venkateswar Venkataraman, Anuradha Krishnan and Rameshwar K. Sharma

The Unit of Regulatory and Molecular Biology, Departments of Cell Biology and Ophthalmology, SOM \& NJMS, University of Medicine and Dentistry of New Jersey, Stratford, NJ, USA

Mol Cell Biochem 267: 107-122, 2004

Due to a technical error Figure 8 of the above article was printed in black \& white.

The figure is herewith reprinted in color. 


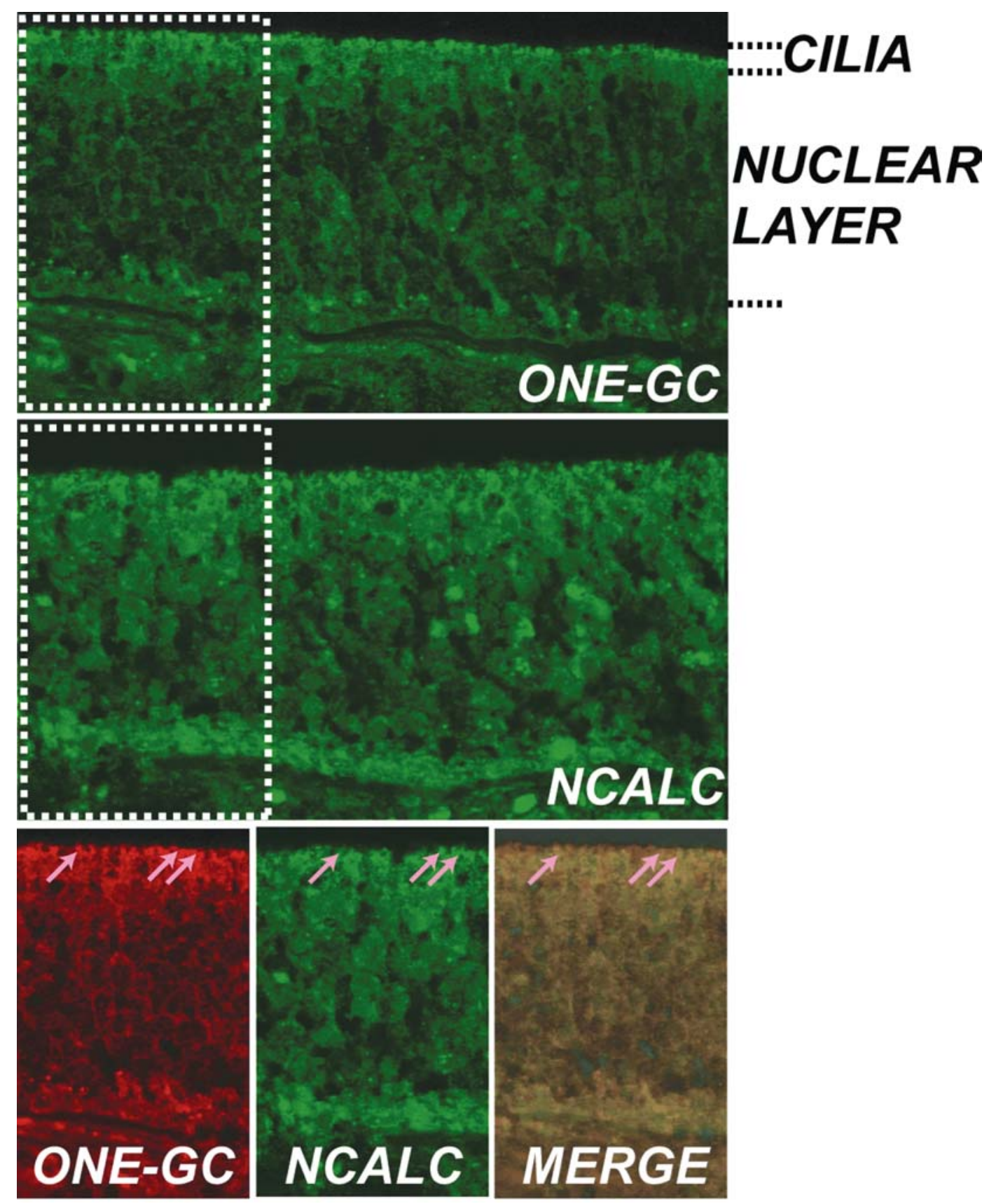

Fig. 8. ONE-GC and neurocalcin $\delta$ in the olfactory neuroepithelium - immunohistochemical analysis. Consecutive cryosections of olfactory neuroepithelium were immunostained for ONE-GC and neurocalcin $\delta$ and the results are presented in the respective panels: "ONE-GC" and "NCALC." Positive staining is in green. The cilia and nuclear layers have been indicated in the top, ONE-GC, panel. The bottom panel presents a composite. Boxed regions from the ONE-GC and NCALC panels have been cropped, the "ONE-GC" panel has been pseudo-colored (red) and overlapped with the NCALC panel (staining in green) using ImagePro software. The result is presented in the "MERGE" panel. In this panel, ONE-GC staining is in red, neurocalcin $\delta$ staining is in green and the regions where they are co-localized are in orange-yellow. Three such regions of the cilia have been indicated by purple arrows. 